\title{
Image and Diagnosis
}

\section{Kawasaki Disease: Opthalmology and Coronary Artery Complications \\ S Williams-Phillips}

\section{Clinical data}

The index case is a 6 years old Afro-caribbean female who had non-specific symptoms of low grade fever, macular popular rash in first week of illness with no subungual peeling or Beau's lines and general feeling of malaise and lethargy for two weeks. She complained in the $3^{\text {rd }}$ week of poor vision in left eye whilst reading with reduced left visual acuity on examination, which was the only significant finding on examination in a non-immunocompromised child with normal growth parameters. Of negative significance she had no dysrhythmias, cardiac murmurs nor signs of heart failure.

Haematological tests showed an increase in platelet count, Chest X-Ray and Electrocardiogram were normal. Ophthalmological assessment confirmed impaired visual acuity in left eye. Transthoracic Echocardiography confirmed Kawasaki Disease with dilated origin of left coronary artery with diameter of $0.416 \mathrm{~cm}$ (Fig. 1), dilated distal left main coronary artery aneurysm with diameter of $0.486 \mathrm{~cm}$ and dilated anomalous origin right coronary artery with diameter $0.400 \mathrm{~cm}$, from one o'clock position (Fig. 3).

From: TAI Wing, Andrews Memorial Hospital, Kingston, Jamaica, West Indies.

Correspondence: Dr S Williams-Phillips, Andrews Memorial Hospital, TAI Wing 27 Hope Road, Kingston 10, Jamaica, West Indies

E-mail: sandrap@cwjamaica.com. 


\section{Diagnosis}

Kawasaki disease is an acute, self-limited small and medium sized vasculitic syndrome affecting multiple body systems with life-threatening panvasculitis of coronary arteries with thrombosis of coronary arteries, coronary artery aneurysms, coronary stenosis, dysrhythmias, heart failure and myocardial infarction which is the most common cause of death in Kawasaki disease. The disease occurs in three phases; acute febrile (0 to 19 Days), subacute (12 to 25 Days), and late or convalescent ( 6 to 8 Weeks), $80 \%$ in infants and young children under 5 years of age with a $50 \%$ peak at 18 months. Diagnosis, based on clinical criteria can be enigmatic and present with an "Atypical Kawasaki" illnesses that does not fulfil the standard diagnostic criteria, as occurred in this Index case, but still have the coronary artery disease development. Conjunctivitis and ophthalmology complications are seen in the acute febrile few days of the illness, are usually painless and not associated with exudate or oedema. The focused feature of the index case are the transthoracic images, which confirmed the diagnosis of Kawasaki. Nonspecific thrombocytosis provided the haematological clue. The anomalous origin of right coronary artery, from one o'clock position, is of no clinical significance.

\section{Comment}

Kawasaki disease, treated with intravenous gamma globulin (IVIG) in the acute phase within the first ten days, of the disease have fewer than 5\% develop coronary artery abnormalities versus the untreated who have $20 \%$ involvement, with incidence of death in $1 \%$ from Myocardial Infarction $(1,2)$. Other modalities of therapy are Aspirin, and if deemed necessary; Tumour necrosis factor and plasmapheresis (1). 
There are no specific diagnostic tests for Kawasaki disease; however, several abnormalities have been identified including thrombocytosis $(1,2,3)$.

Electrocardiogram (ECG) can show the electrical conduction of the myocardium which may vary, with pancarditis, and have flat, depressed ST segment or signs of myocardial ischaemia (1).

Transthoracic echocardiography is the main and primary diagnostic modality. Cardiac abnormalities in the acute phase include tachycardia, dysrhythmias, hyperdynamic precordium, gallop rhythm, myocarditis, depressed myocardial contractility, mitral regurgitation, and coronary artery abnormalities $(1,2,3)$.

In the subacute phase they are afebrile but panvasculitis of coronary arteries, inflammation, aneurysms, thrombosis formation, is occurring and can lead to stenosis or obstruction leading to congestive heart failure and myocardial infarction $(1,2,3)$.

Hence it requires a high index of suspicion by Paediatricians and Cardiologists, to facilitate diagnosis in the acute phase, before the subacute and the convalescent phase, where they are afebrile, with remission of the signs of the illness and return to premorbid personality and energy level. 


\section{REFERENCES}

1. Gedalia A1. Kawasaki disease: an update. Curr Rheumatol Rep 2002; 4: 25-9.

2. Oates-Whitehead RM, Baumer JH, Haines L, Love S, Maconochie IK, Gupta A, Roman 3.

3. K, Dua JS, Flynn I. Intravenous immunoglobulin for the treatment of Kawasaki disease in children. DOI: 10.1002/14651858.CD004000

4. Cochrane Database Syst Rev 2003; CD004000.

5. Treatment of acute Kawasaki disease: aspirin's role in the febrile stage revisited.

6. Hsieh KS, Weng KP, Lin CC, Huang TC, Lee CL, Huang SM. Treatment of acute Kawasaki disease: aspirin's role in the febrile stage revisited. Pediatrics. 2004 Dec; 14:e689-93. 


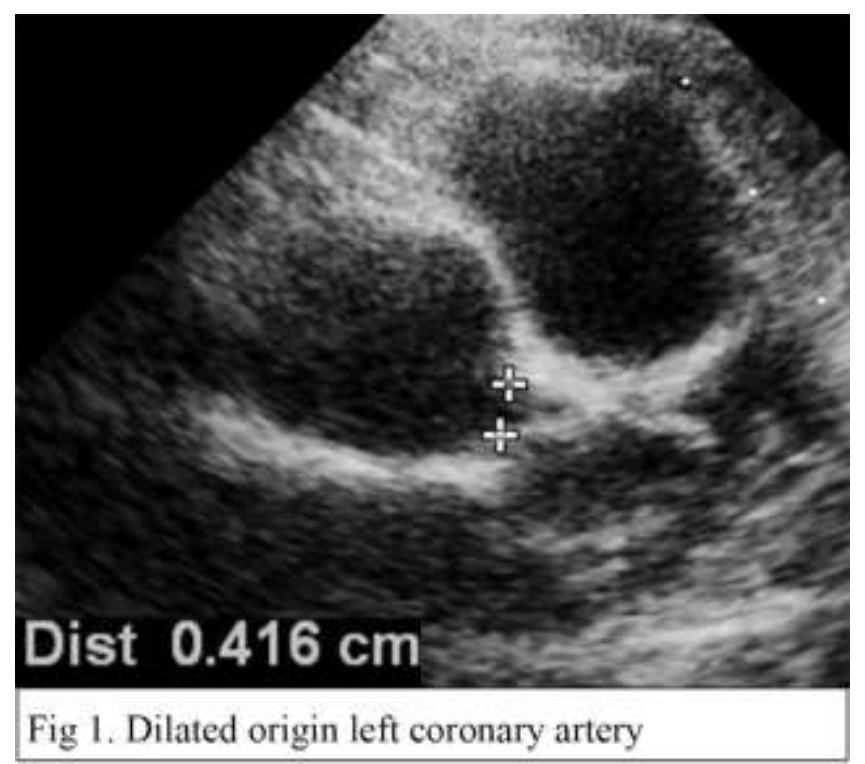

Fig. 1. Dilated origin of left coronary artery. Dist $0.416 \mathrm{~cm}$.

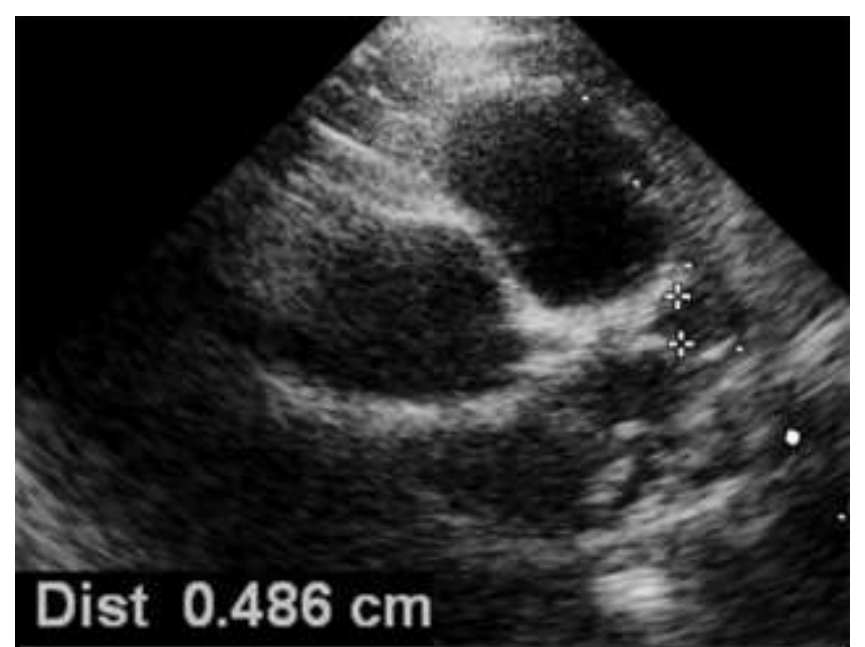

Fig 2. Dilated distal left coronary artery (aneurysm)

Fig. 2. Dilated distal left coronary artery (aneurysm) Dist 0.486. 
Kawasaki Coronary Disease and Ophthalmology

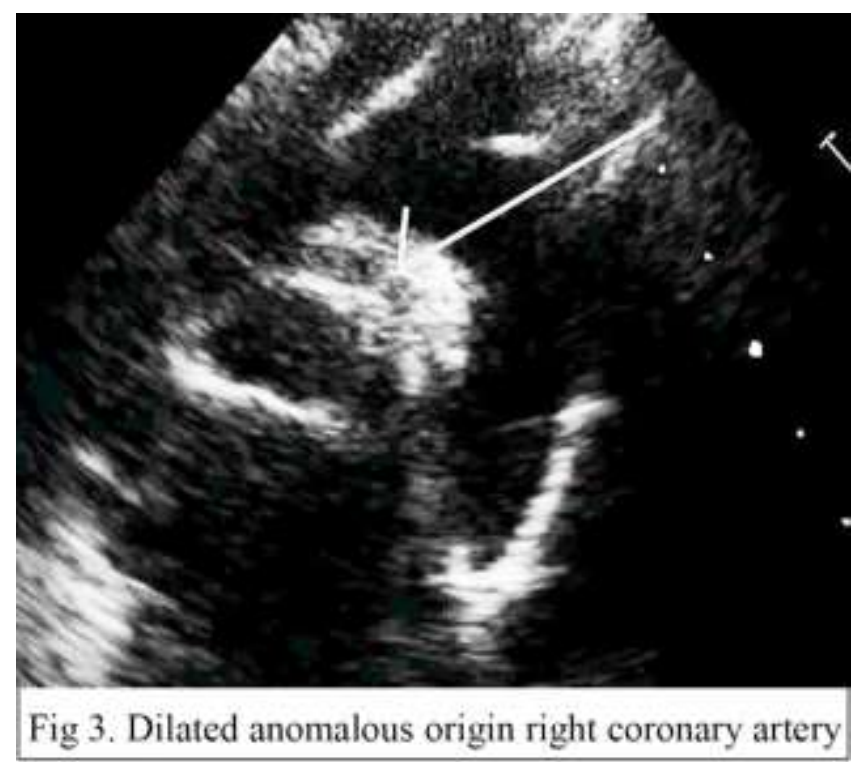

Fig.3. Dilated anomalous origin of right coronary artery. $0.40 \mathrm{~cm}$. 